\title{
Zn Electrodeposition on Single-Crystal GaN(0001) Surface: Nucleation and Growth Mechanism
}

\author{
Fei Peng, ${ }^{1,2}$ Shuang-Jiao Qin, ${ }^{2}$ Yu Zhao, ${ }^{2}$ and Ge-Bo Pan ${ }^{2}$ \\ ${ }^{1}$ Nano Science and Technology Institute, University of Science and Technology of China, Suzhou 215123, China \\ ${ }^{2}$ Suzhou Institute of Nano-Tech and Nano-Bionics, Chinese Academy of Sciences, Suzhou 215123, China
}

Correspondence should be addressed to Ge-Bo Pan; gbpan2008@sinano.ac.cn

Received 30 September 2015; Revised 13 January 2016; Accepted 14 January 2016

Academic Editor: Sheng S. Zhang

Copyright (C) 2016 Fei Peng et al. This is an open access article distributed under the Creative Commons Attribution License, which permits unrestricted use, distribution, and reproduction in any medium, provided the original work is properly cited.

The electrochemical deposition of zinc on single-crystal $n$-type GaN(0001) from a sulphate solution has been investigated on the basis of electrochemical techniques including cyclic voltammetry, chronoamperometry, and Tafel plot. The morphology and crystal structure of zinc deposits have been characterized by means of scanning electron microscopy, X-ray diffraction, and energydispersive X-ray analysis. The result has revealed that the deposition of $\mathrm{Zn}$ on GaN electrode commenced at a potential of -1.12 V versus $\mathrm{Ag} / \mathrm{AgCl}$. According to the Tafel plot, an exchange current density of $\sim 0.132 \mathrm{~mA} \mathrm{~cm}^{-2}$ was calculated. In addition, the current transient measurements have shown that $\mathrm{Zn}$ deposition process followed the instantaneous nucleation in $10 \mathrm{mM} \mathrm{ZnSO}_{4}+0.5 \mathrm{M}$ $\mathrm{Na}_{2} \mathrm{SO}_{4}+0.5 \mathrm{M} \mathrm{H}_{3} \mathrm{BO}_{3}(\mathrm{pH}=4)$.

\section{Introduction}

The electrodeposition of zinc $(\mathrm{Zn})$ and its alloys are of practical and industrial importance due to their excellent anticorrosive properties [1-5]. Diverse factors, such as $\mathrm{pH}$ [6], concentration of $\mathrm{Zn}$ ions [7], anions [8], and organic additives [9], are found to influence the $\mathrm{Zn}$ electrodeposition. However, it is noted that previous studies of $\mathrm{Zn}$ electrodeposition are mostly performed on metal electrodes (e.g., steel [5], nickel [10], platinum [11], copper [12], gold [13], and aluminum [14]). To the best of our knowledge, no report has described the $\mathrm{Zn}$ electrodeposition on semiconductor electrodes although they are of interest in modern technologies, such as lightemitting diodes and microelectromechanical systems [1517]. This is due to complicated thermodynamics and kinetics at metal/semiconductor interface, induced by unique charge transfer, band structure, and weak metal-substrate interaction.

On the other hand, gallium nitride- (GaN-) based materials, with a wide band gap, have recently attracted great attention because of their wide applications in advanced optoelectronic devices [18-20]. Besides physics applications, there is an increasing interest in electrochemistry field due to their unique physicochemical properties. For instance, Pt nanoparticle-modified $\mathrm{GaN}$ was found to have potential applications in catalysis and sensors $[21,22]$. Pd-S/GaN was a very efficient and recycled green catalyst for Heck reaction [23]. We have demonstrated that $\mathrm{GaN}$ might be an elegant alternative catalyst support similar to $\mathrm{WO}_{3}, \mathrm{TiO}_{2}$, and $\mathrm{ZnO}$ [24-26]. They have shown excellent chemical stability, large potential window for water electrolysis, good electric conductivity, and low background current.

Herein, a study of the electrodeposition of $\mathrm{Zn}$ on single-crystal $n$-type GaN(0001) electrode is presented. The electrochemical reduction of $\mathrm{Zn}^{2+}$ from sulphate solution was investigated by basic electrochemical analysis including cyclic voltammetry (CV), chronoamperometry (CA), and Tafel plot. The morphology and crystal structure of $\mathrm{Zn}$ deposits were characterized by means of scanning electron microscopy (SEM), X-ray diffraction (XRD), and energydispersive $\mathrm{X}$-ray analysis (EDAX).

\section{Material and Methods}

Single-crystal $n$-type GaN(0001) substrates were grown on sapphire(0001) by a hydride vapor phase epitaxy. The GaN 
layer was $5 \mu \mathrm{m}$ thick, Si-doped, and with a carrier concentration of $4 \times 10^{18} \mathrm{~cm}^{-3}$. Before each experiment, the working electrode of $\mathrm{GaN}$ was cleaned with ultrasonication in acetone, ethanol, and distilled water successively for $15 \mathrm{~min}$. The Pt wires $(1 \mathrm{~mm}$ in diameter) were used as counter electrodes.

Zinc sulphate $\left(\mathrm{ZnSO}_{4}\right)$, boric acid $\left(\mathrm{H}_{3} \mathrm{BO}_{3}\right)$, sodium sulphate $\left(\mathrm{Na}_{2} \mathrm{SO}_{4}\right)$, ethanol, and acetone were of analytical grade and purchased from Sinopharm Chemical Reagent Co., Ltd. The electrolyte used in the present study was $10 \mathrm{mM} \mathrm{ZnSO}_{4}+0.5 \mathrm{M} \mathrm{H}_{3} \mathrm{BO}_{3}+0.5 \mathrm{M} \mathrm{Na}_{2} \mathrm{SO}_{4}$ if it was not mentioned. All the solutions were prepared by using Milli-Q water $(>18 \mathrm{M} \Omega)$ and all the reagents were used as received.

The electrochemical experiments used a standard threeelectrode cell arrangement and were undertaken with a CHI 660D potentiostat/galvanostat (Shanghai ChenHua Co., Ltd.). The distance between working and counter electrodes was about $1 \mathrm{~cm}$. The geometric area of $\mathrm{GaN}$ in contact with the electrolyte was $0.5 \mathrm{~cm}^{2}$. All the potentials were reported with respect to a saturated $\mathrm{Ag} / \mathrm{AgCl}$ electrode ( $0.197 \mathrm{~V}$ versus NHE).

The morphology of $\mathrm{Zn}$ deposits was investigated by scanning electron microscopy (Hitachi S4800). The X-ray diffraction (XRD) patterns were recorded on a Bruker D8 Advance Powder X-Ray Diffractometer at a scanning rate of $0.02^{\circ} \mathrm{s}^{-1}$ in the $2 \theta$ range 20 to $80^{\circ}$ using $\mathrm{Cu}-\mathrm{Ka}$ radiation $(\lambda=1.5406 \AA)$. The energy-dispersive X-ray analysis (EDAX) was performed on a Quanta 400 FEG SEM at $10 \mathrm{KV}$.

\section{Results and Discussion}

In principle, electrochemical nucleation involves the diffusion and charge transfer across the electrolyte/electrode interface. In comparison with metal electrodes, the deposition on semiconductor electrodes is more complicated. This is mainly due to the unique band structure of semiconductors, which affects both thermodynamics and kinetics of deposition processes. The existence of $\mathrm{Zn}(\Pi)$ in $0.5 \mathrm{M} \mathrm{Na}_{2} \mathrm{SO}_{4}$ and $0.5 \mathrm{M}$ $\mathrm{H}_{3} \mathrm{BO}_{3}$ solution is generally considered to be $\mathrm{Zn}^{2+}$ due to its stability. The reduction process of $\mathrm{Zn}^{2+}$ can be expressed by

$$
\mathrm{Zn}^{2+}+2 \mathrm{e}^{-}=\mathrm{Zn} \text {. }
$$

The Femi level is close to the conduction band due to the high doped concentration. Figure 1 shows the energy band diagram of the $n$-type GaN/electrolyte interface. It can be seen that there are two sections. For simplicity, the redox couple is considered as energy states of the Gerischer model of energy levels in the right side. The midpoint between the reduction and oxidation states corresponds to the equilibrium potential, $E_{\mathrm{O} / \mathrm{R}}$, which is equivalent to a Fermi level of a redox system:

$$
E_{\mathrm{O} / \mathrm{R}}=E_{\mathrm{O} / \mathrm{R}}^{\theta}+k T \ln \left(\frac{c_{\mathrm{OX}}}{c_{\mathrm{red}}}\right) .
$$

The band energy of GaN was cited from the literature [27]. When $\mathrm{GaN}$ was in contact with electrolyte, the band energy would bend upward. More importantly, the Fermi energy of semiconductor and solution is equal at the electrostatic

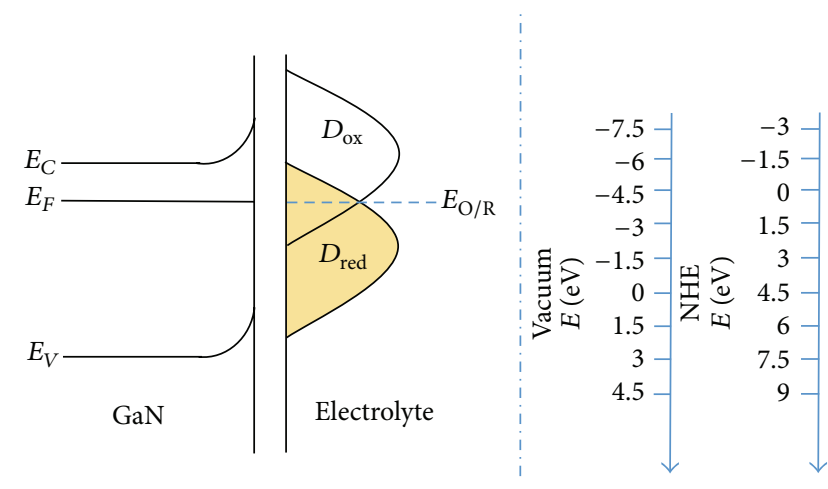

FIGURE 1: The energy band diagram for the $n$-type GaN/electrolyte interface.

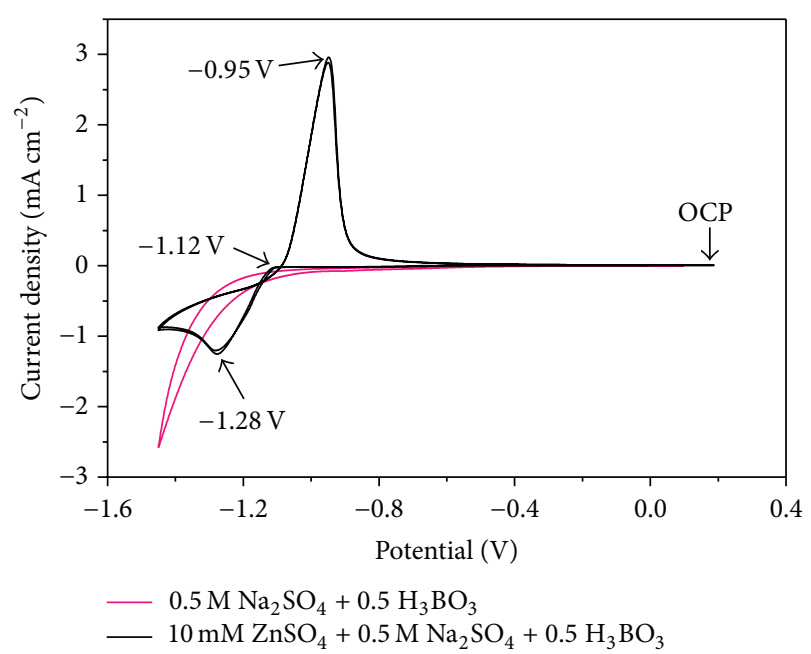

FIGURE 2: Cyclic voltammograms of GaN electrode at the scan rate of $10 \mathrm{mV} \mathrm{s}^{-1}$.

equilibrium condition. As a result, a space charge layer (SCL) was formed at the interface between semiconductor and solution, which is equivalent to the built-in potential formed at a Schottky junction [27].

Figure 2 presents typical CVs of $\mathrm{GaN}$ electrode in $0.5 \mathrm{M}$ $\mathrm{H}_{3} \mathrm{BO}_{3}+0.5 \mathrm{M} \mathrm{Na}_{2} \mathrm{SO}_{4}$ solution with and without $10 \mathrm{mM}$ $\mathrm{ZnSO}_{4}$ at a scan rate of $10 \mathrm{mV} / \mathrm{s}$. The Open Circuit Potential (OCP) was about $0.18 \mathrm{~V}$. In the first cycle, the cathodic current started to increase when the potential reached $-1.12 \mathrm{~V}$, indicating the beginning of $\mathrm{Zn}$ deposition. Moreover, an obvious cathodic reduction peak at potential of $-1.28 \mathrm{~V}$ can be observed. As described in the literatures $[28,29]$, the reduction proceeds in a single step (peak at $-1.28 \mathrm{~V}$ ) associated with the reduction of $\mathrm{Zn}(\mathrm{II})$ ions to $\mathrm{Zn}(0)$. No additional peaks are observed before overpotential deposition of $\mathrm{Zn}$, indicating that no underpotential process occurs. This is mainly due to the weak interaction between $\mathrm{Zn}$ deposit and $\mathrm{GaN}$. On the reverse scan, the anodic stripping peak is observed at a potential of $-0.95 \mathrm{~V}$ due to the dissolution of $\mathrm{Zn}$. In the second cycle, the $\mathrm{Zn}$ deposition on GaN electrode commenced more positive than that of first one because some Zn nuclei were still present on the electrode surface. 


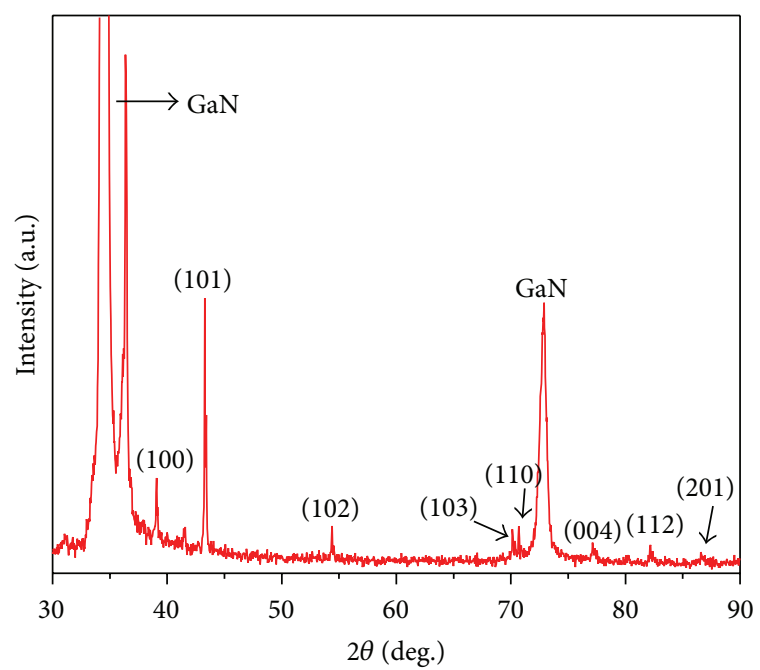

(a)

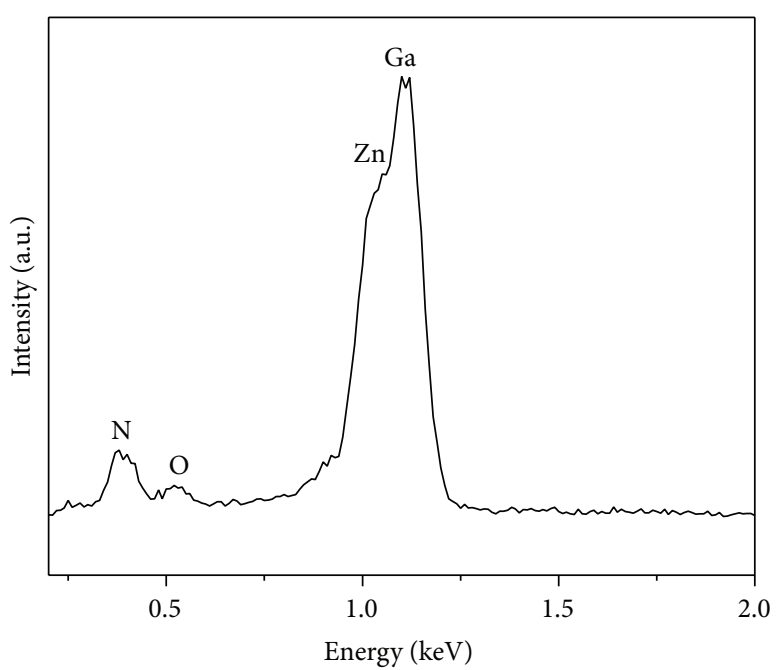

(b)

Figure 3: (a) X-ray patterns and (b) EDAX spectra of Zn deposits.

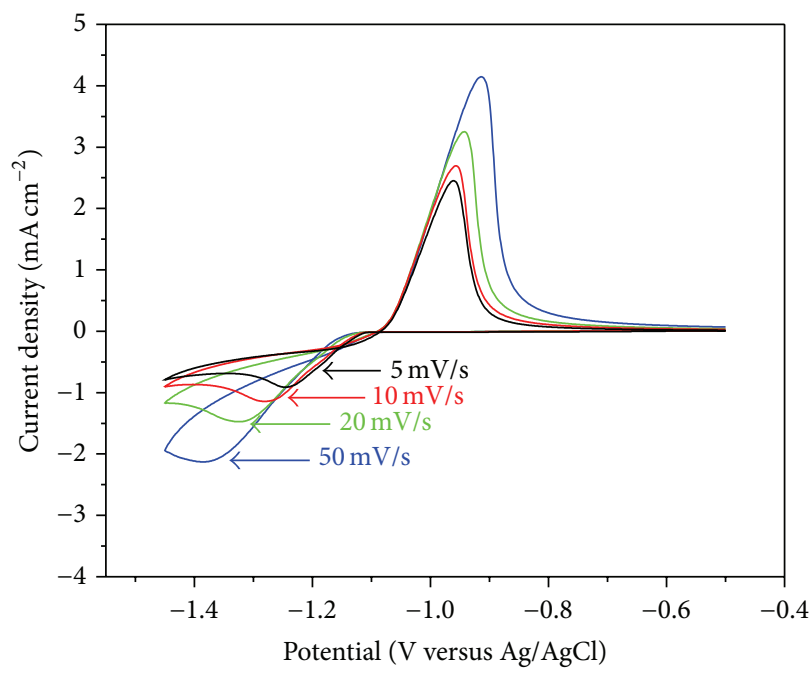

(a)

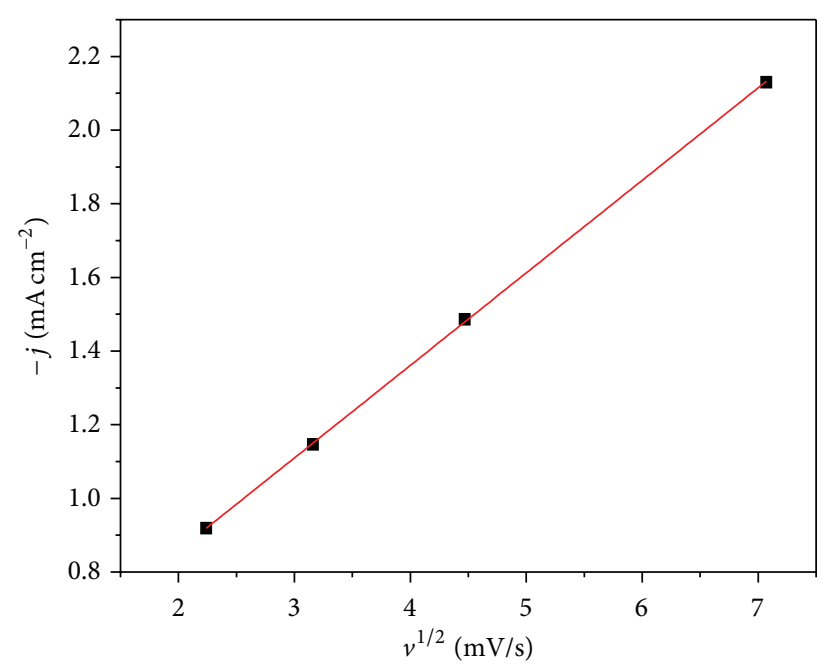

(b)

Figure 4: (a) Cyclic voltammograms for Zn deposition on GaN at different scan rates. (b) A fitted line plot for the current density of cathodic peak versus the square root of the scan rate.

Figure 3(a) displays the XRD patterns of $\mathrm{Zn}$ deposits on $\mathrm{GaN}$. The deposition potential is $-1.4 \mathrm{~V}$, and the deposition time is $7200 \mathrm{~s}$. The thickness of the $\mathrm{Zn}$ deposits is about $400 \mathrm{~nm}$. The XRD pattern revealed five characteristic peaks at $2 \theta=38.9^{\circ}, 43.2^{\circ}, 54.3^{\circ}, 70.1^{\circ}$, and $70.6^{\circ}$, which are attributed to the $\mathrm{Zn}$ diffraction peaks of the (100), (101), (102), (103), and (110) planes (JCPDS Card Number 87-0713), respectively. The crystals grew predominantly with a (101) crystallographic orientation. This is consistent with previous references [28]. Figure 3(b) shows the EDAX spectra of $\mathrm{Zn}$ deposits on GaN. The EDAX spectra obtained in local points of the electrodeposits surface showed that they were mainly composed by $\mathrm{Zn}$. Gallium and nitrogen were also detected due to the GaN substrate. The detected oxygen may be associated with the oxidation of a little part of $\mathrm{Zn}(0)$ in the solution or air.

Figure 4(a) shows a family of CVs of Zn deposited on GaN at different sweep rates. With the decrease of sweep rate, the $\mathrm{Zn}$ deposition commences at more positive potential and the peak current increases. Figure 4(b) shows a linear variation of the cathodic peak current density as a function of the square root of the scan rate $\left(v^{1 / 2}\right)$. According to Berzins-Delahay equation (3), this linear relation supports the suggestion that the growth of zinc metal is a diffusion-controlled process [30]:

$$
i_{p}=\frac{0.6106(n F)^{3 / 2}}{C_{j}(R T)^{1 / 2} D_{j}^{1 / 2} v^{1 / 2}}
$$




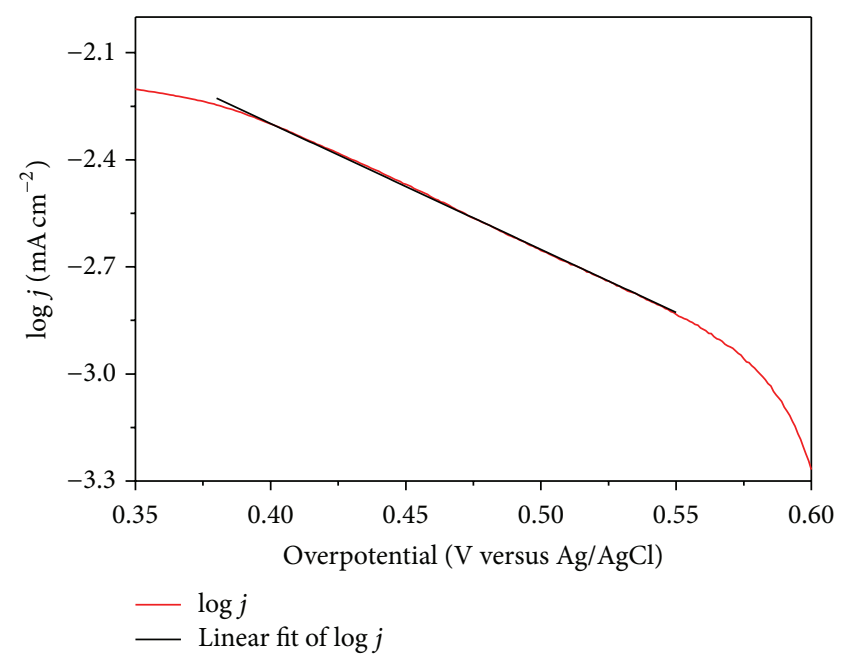

FIgURE 5: Tafel plot for the electrochemical reduction of $\mathrm{Zn}^{2+}$ on $\mathrm{GaN}$ electrode. The scan rate was $10 \mathrm{mV} \mathrm{s}^{-1}$.

Meanwhile, the electrochemical behavior of $\mathrm{Zn}$ on GaN was irreversible. For electron transfer reaction, the cathodic transfer coefficient $(\alpha)$ can be calculated by the following equation [31]:

$$
\left|V_{p}-V_{p / 2}\right|=\frac{0.048}{\alpha}
$$

$V_{p}(\mathrm{~V})$ is the peak potential and $V_{p / 2}(\mathrm{~V})$ is the potential at half peak height. The peak potential depends on the sweep rate, and the number of $\alpha$ is 0.53 at a sweep rate of $10 \mathrm{mV} \mathrm{s}^{-1}$. The value of cathodic transfer coefficient is slightly equal to anodic transfer coefficient $(1-\alpha)$.

It is well known that the electrode kinetics can be simply described by the Butler-Volmer equation:

$$
j=j_{0}\left\{\exp \left[(1-\alpha) \frac{z F}{R T} \eta\right]-\exp \left[-\alpha \frac{z F}{R T} \eta\right]\right\},
$$

where $j\left(\mathrm{~mA} \mathrm{~cm}^{-2}\right)$ presents the current density, $j_{0}\left(\mathrm{~mA} \mathrm{~cm}^{-2}\right)$ is the exchange current density, and $\eta(\mathrm{V})$ is the overpotential. For large cathodic overpotentials $(|\eta|>0.1 \mathrm{~V})$, the anodic branch is very small and can be neglected. The resulting Tafel law corresponding to electrochemical reaction of $\mathrm{Zn}^{2+}$ can then be written as

$$
\ln |j|=\ln j_{0}-\alpha \frac{z F}{R T} \eta \text {. }
$$

Figure 5 illustrates the typical Tafel plot for the electrochemical reduction of $\mathrm{Zn}^{2+}$ on $\mathrm{GaN}$ electrode. It can be seen that a portion of the curve exhibits a linear relationship in the plot of $\log j$ versus potential. This indicates that the mass transport is the dominant rate-limiting step [28]. In addition, the cathodic exchange current density is estimated to be $\sim 0.132 \mathrm{~mA} \mathrm{~cm}^{-2}$, which is only slightly lower than those on other substrates [7]. This is mainly because the number of free electrons in the conduction band of $\mathrm{GaN}$ is lower than metal.

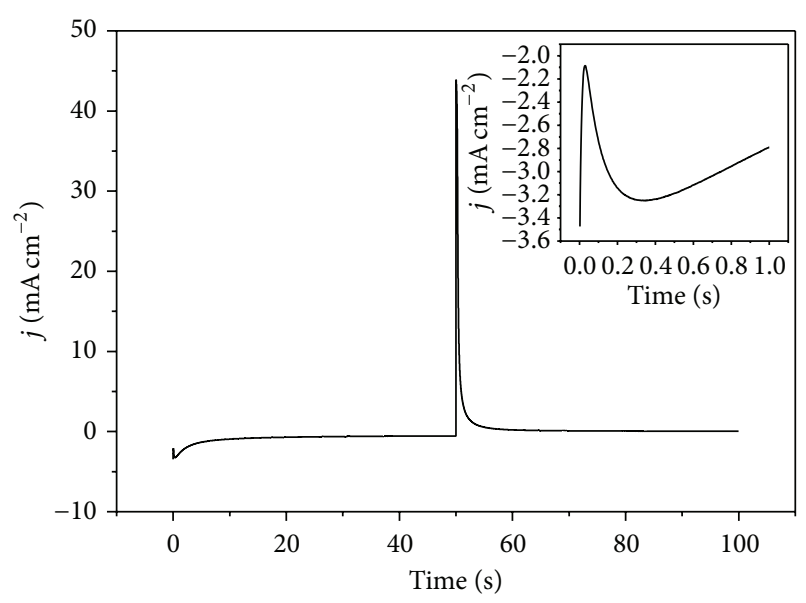

(a)

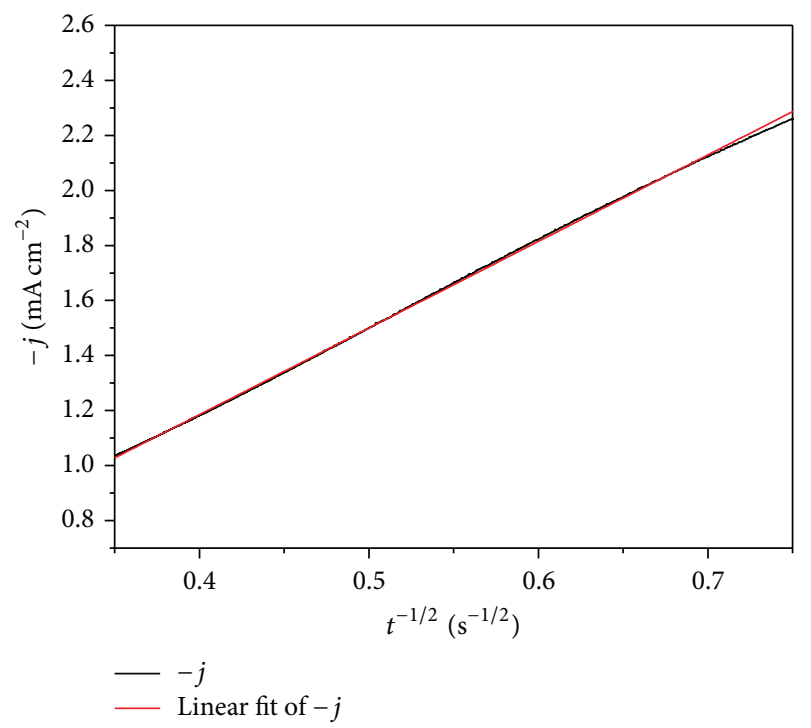

(b)

Figure 6: (a) Potentiostatic current transient of $\mathrm{Zn}$ deposition on $\mathrm{GaN}$; the inset shows an enlargement of the transient curve for the first seconds; (b) Cottrell plot for the determination of the diffusion coefficient of $\mathrm{Zn}^{2+}$ species.

According to the Cottrell equation, diffusion of electroactive species in solution could be quantitatively described from the diffusion coefficient $\left(D_{j}, \mathrm{~cm}^{2} \mathrm{~s}^{-1}\right)$ :

$$
|j|=n F A D_{j}^{1 / 2} C_{j} \pi^{-1 / 2} t^{-1 / 2} .
$$

$j$ (A) is the cathodic current density, $n$ is the number of electrons exchanged, and $A\left(\mathrm{~cm}^{2}\right)$ is the effective area of the electrode. $D_{j}$ represents the diffusion coefficient and $C_{j}$ is the concentration of $\mathrm{Zn}^{2+}$. Before the occurrence of the limiting current, by plotting $|j|=f\left(t^{-1 / 2}\right)$, a linear graph would be obtained until the growing diffusion layer has reached its maximum width.

Figure 6(a) shows potentiostatic current transients of the electrodeposition and oxidation process for the $\mathrm{Zn}$ on $\mathrm{GaN}$ substrate. The deposition potential is $-1.4 \mathrm{~V}$, while the 
reverse pulse is maintained constant at $-0.15 \mathrm{~V}$, and the pulse time is $50 \mathrm{~s}$. The resulting curves of the transients are a typical response of an electrochemical nucleation and growth process. The inset shows an enlargement of the transient curve for the first seconds. It is clear that the nucleation stage lasts about $0.3 \mathrm{~s}$. The rapid increase in current at very short times indicates the growth of the new phase and the increasing number of nuclei present on the electrode surface. As these grow, the coalescence of neighboring diffusion fields with localized spherical symmetry gives rise to a current maximum, followed by a decaying current, related to planar electrode diffusion. It decreases asymptotically until reaching a constant current value. Furthermore, the charges associated with the reduction and oxidation processes, $Q_{C}$ and $Q_{A}$, were obtained by integrating the cathodic and the anodic branches of the current transients. The values of $Q_{C}$ and $Q_{A}$ were $41.95 \mathrm{~mA}$ and $29.83 \mathrm{~mA}$, respectively. That is, the efficiency $\left(Q_{A} / Q_{C}\right)$ of $\mathrm{Zn}$ ions reduction on the GaN electrode was 0.689. This further confirmed that the electrochemical behavior of $\mathrm{Zn}$ on $\mathrm{GaN}$ was irreversible although the contribution of hydrogen reduction to the $Q_{C}$ cannot be excluded completely. The linear part of the experimental Cottrell plot can be seen in Figure 6(b) as well as the corresponding data fit. The diffusion coefficient $\left(D_{j}\right)$ was evaluated by analyzing the experimental data that corresponds to the fall of the direct pulse transients. The diffusion coefficient of $D_{j}=3.33 \times 10^{-5} \mathrm{~cm}^{2} \mathrm{~s}^{-1}$ was found for $\mathrm{Zn}^{2+}$ species. This value is comparable to the literatures $[4,28,29]$.

The electrocrystallization process of $\mathrm{Zn}$ is limited by diffusion, thus the transients in Figure 5 can be analyzed through a $3 \mathrm{D}$ growth for an instantaneous nucleation:

$$
j=\frac{z F D_{j}^{1 / 2}}{\pi^{1 / 2} t^{1 / 2}}\left[1-\exp \left(-N_{0} \pi t K D_{j}\right)\right],
$$

or for a progressive type nucleation:

$$
j=\frac{z F D_{j}^{1 / 2} C_{j}}{\pi^{1 / 2} t^{1 / 2}}\left[1-\exp \left(\frac{-2 A N_{0} \pi t^{2} K D_{j}}{3}\right)\right] .
$$

$N_{0}\left(\mathrm{~cm}^{-2}\right)$ is number density of nucleation active sites, $A\left(\mathrm{~s}^{-1}\right)$ is the steady-state nucleation rate active site, and $K$ is the nondimensional growth rate constant of a nucleus, defined as $K=\left(8 \pi C_{j} M / \rho\right)^{1 / 2}$. Table 1 shows the values of nucleation kinetic parameter Ns for different concentration and potential.

The experimental values of $j_{m}$ and $t_{m}$ could be obtained from the potentiostatic transients, and theoretical maximum values can be evaluated by the following equations, for the instantaneous case:

$$
\begin{aligned}
& t_{m}=\frac{1.2564}{N_{0} \pi K D_{j}}, \\
& \mathrm{Ns}=\frac{1.2564}{t_{m} \pi K D_{j}}, \\
& j_{m}=0.6382 z F D_{j} C_{j}\left(K N_{0}\right)^{1 / 2},
\end{aligned}
$$

TABLE 1: The values of nucleation kinetic parameter Ns for each concentration and potential.

\begin{tabular}{lccc}
\hline Potential/V $C_{j}\left(\mathrm{Zn}^{2+}\right) / \mathrm{mol} \mathrm{cm}^{-3}$ & $\begin{array}{c}\mathrm{Ns} \\
\text { (instantaneous) }\end{array}$ & $\begin{array}{c}\text { Ns } \\
\text { (progressive) }\end{array}$ \\
\hline-1.25 & $1 \times 10^{-5}$ & $0.78 \times 10^{5}$ & $1.26 \times 10^{5}$ \\
-1.3 & $1 \times 10^{-5}$ & $1.78 \times 10^{5}$ & $1.89 \times 10^{5}$ \\
-1.4 & $1 \times 10^{-5}$ & $2.91 \times 10^{5}$ & $4.71 \times 10^{5}$ \\
-1.4 & $5 \times 10^{-6}$ & $1.97 \times 10^{5}$ & $3.18 \times 10^{5}$ \\
-1.4 & $2 \times 10^{-5}$ & $2.25 \times 10^{5}$ & $3.64 \times 10^{5}$ \\
\hline
\end{tabular}

for the progressive case:

$$
\begin{aligned}
& t_{m}=\left(\frac{4.6733}{\pi A N_{0} K D_{j}}\right)^{1 / 2}, \\
& \mathrm{Ns}=\left(\frac{A N_{0}}{2 K D_{j}}\right)^{1 / 2}, \\
& j_{m}=0.4615 z F D_{j}^{3 / 4} C_{j}\left(K A N_{0}\right)^{1 / 4} .
\end{aligned}
$$

Thus the nondimensional (8) and (9) for the instantaneous case are

$$
\left(\frac{j}{j_{m}}\right)^{2}=\frac{1.9542}{t / t_{m}}\left\{1-\exp \left[-1.2564\left(\frac{t}{t_{m}}\right)\right]\right\}^{2}
$$

and for the progressive case are

$$
\left(\frac{j}{j_{m}}\right)^{2}=\frac{1.2254}{t / t_{m}}\left\{1-\exp \left[-2.3367\left(\frac{t}{t_{m}}\right)^{2}\right]\right\}^{2} \text {. }
$$

Figure 7(a) illustrates current transients for $\mathrm{Zn}$ deposition onto $\mathrm{GaN}$ electrode within potential range from $1.25 \mathrm{~V}$ to $1.4 \mathrm{~V}$. Regardless of the electrode potential all the curves have similar shape and are characterized by sharp increase of cathodic current at the initial stages of deposition followed by a drop at longer times. These features of current transients are relevant to $3 \mathrm{D} \mathrm{Zn}$ nucleation followed by diffusion limited growth. Figure 7(b) shows the normalized cathodic transients from Figure 7 (a) together with the theoretical curves for instantaneous and progressive $3 \mathrm{D}$ nucleation and growth based on (12) and (13). Figure 7(c) shows a series of deposition transients for deposition of zinc from the different concentration of $\mathrm{ZnSO}_{4}$ and same potential $(-1.4 \mathrm{~V})$. The black lines of Figures 7 (b) and $7(\mathrm{~d})$ are the theoretical curves for instantaneous and progressive nucleation. From the results in Figure 7(b), in which the solution concentration of $\mathrm{ZnSO}_{4}$ is $10 \mathrm{mM}$, the first half of the nucleation plot has good correlation with the theoretical curve for instantaneous nucleation, while the second half of the nucleation plots transition from progressive to instantaneous nucleation with the potentials increasing from $-1.25 \mathrm{~V}$ (green lines) to $-1.4 \mathrm{~V}$ (red lines). Meanwhile, we can know that the mechanism of $\mathrm{Zn}$ is different at various concentration from Figure $7(\mathrm{~d})$ and the red, green, and blue lines are the $5 \mathrm{mM}, 10 \mathrm{mM}$, and $20 \mathrm{mM}$. The nucleation plots transition is gradually close 


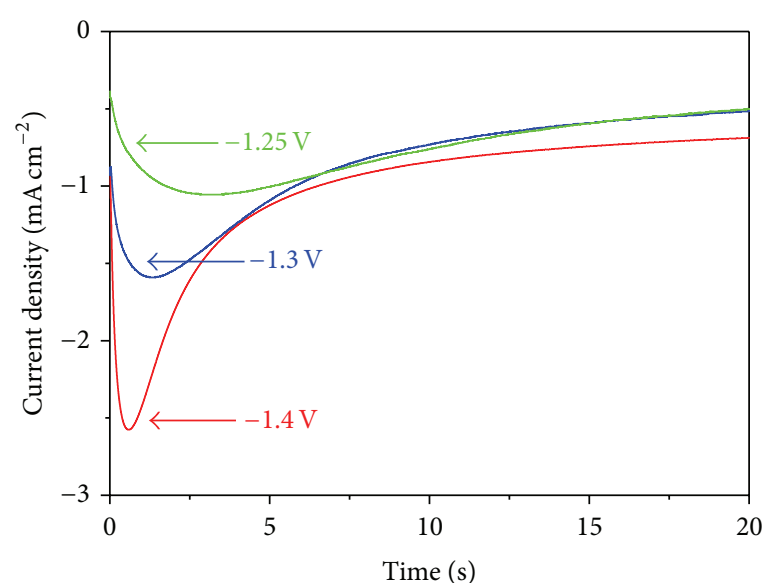

(a)

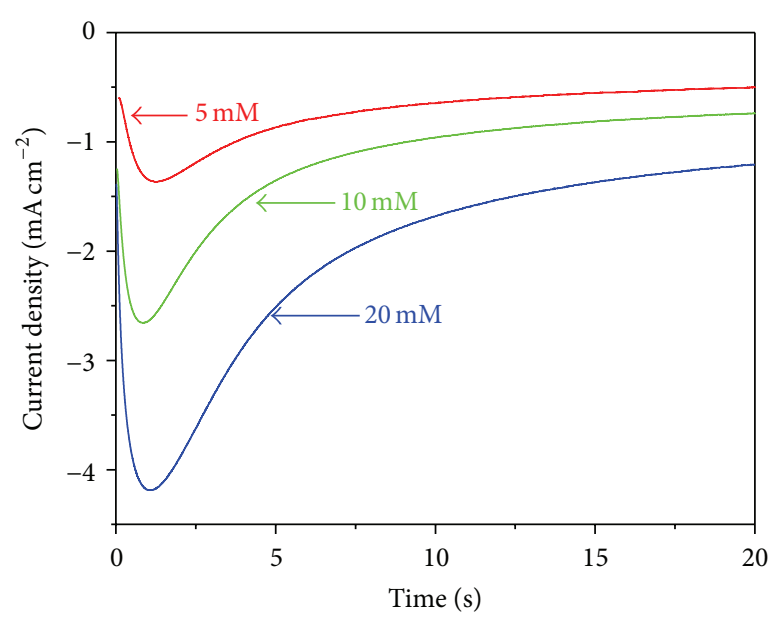

(c)

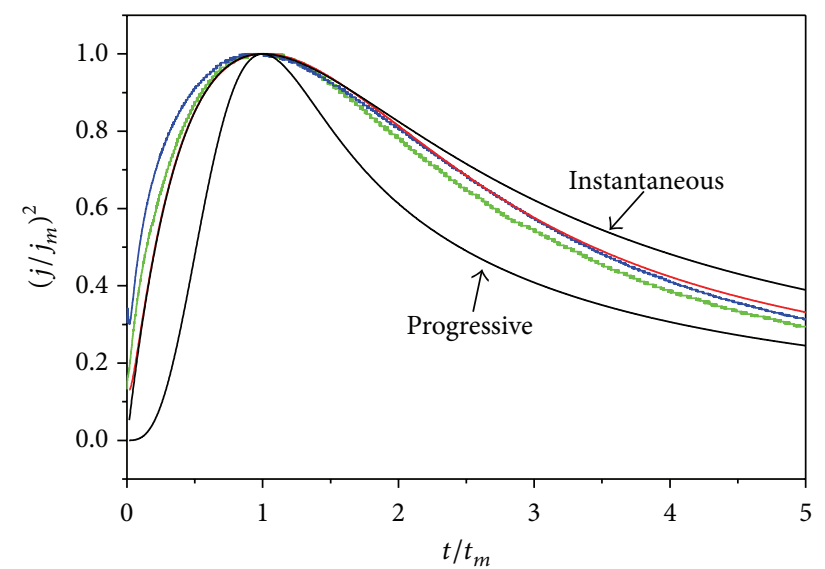

(b)

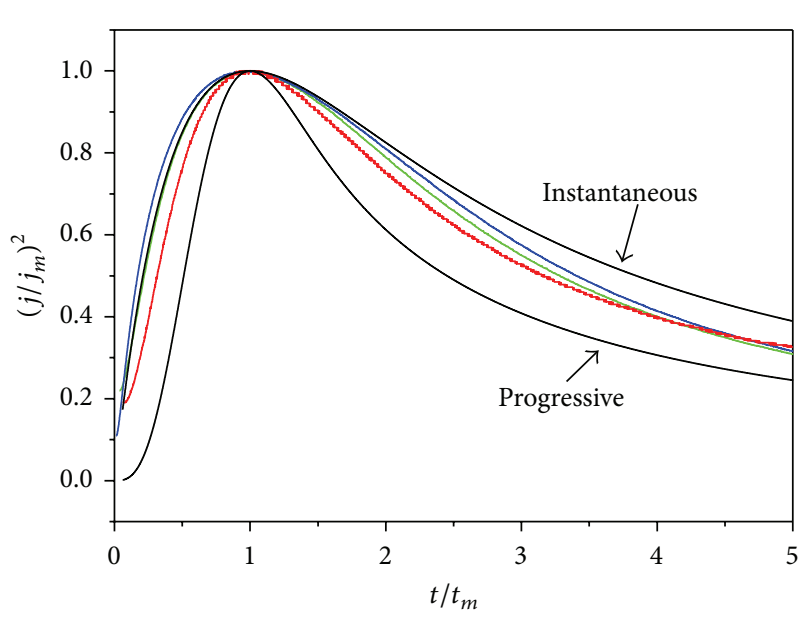

(d)

Figure 7: Potentiostatic current transients for $\mathrm{Zn}$ electrodeposition on $\mathrm{GaN}$. (a) The concentration of $\mathrm{ZnSO}_{4}$ was $10 \mathrm{mM}$ and the potential was varied. (c) The applied potential was $-1.4 \mathrm{~V}$, while the concentration of $\mathrm{ZnSO}_{4}$ was varied. (b) and (d) are the corresponding nondimensional plots of $\left(j / j_{m}\right)^{2}$ versus $\left(t / t_{m}\right)$.

to instantaneous nucleation with increasing $\mathrm{Zn}^{2+}$ concentration.

Figures 8(a)-8(d) show a family of SEM images of Zn deposits on $\mathrm{GaN}$. The applied potential was $-1.4 \mathrm{~V}$ and the deposition time was varied from $10,20,40$, to $80 \mathrm{~s}$. It can be seen that $\mathrm{Zn}$ nuclei were formed on GaN electrode and followed by a 3D island growth (Volmer-Weber) mechanism because of the weak interaction between $\mathrm{GaN}$ and $\mathrm{Zn}$. The nuclei density increased and coalescence occurred when the deposition time was varied from 10 to $80 \mathrm{~s}$.

\section{Conclusion}

The electrodeposition of $\mathrm{Zn}$ on $n$-type single-crystal $\mathrm{GaN}(0001)$ from a sulphate solution has been studied by electrochemical techniques and SEM. Zn deposition on $\mathrm{GaN}$ electrode commenced at a potential of $-1.12 \mathrm{~V}$ without underpotential deposition and was irreversible and masstransfer limited. The deposition occurred on the conduction band of $\mathrm{GaN}$ due to the negative equilibrium potential. Moreover, the exchange current density of $\sim 0.132 \mathrm{~mA} \mathrm{~cm}^{-2}$ was calculated on the basis of Tafel plot. The current transient measurements indicated that the deposition process accorded with the progressive nucleation.

\section{Conflict of Interests}

The authors declare that there is no conflict of interests regarding the publication of this paper.

\section{Acknowledgments}

This work was supported by the National Natural Science Foundation of China (no. 21273272), Key Research 


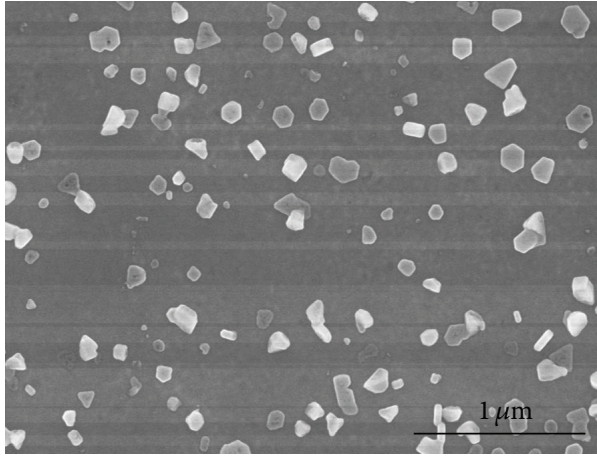

(a)

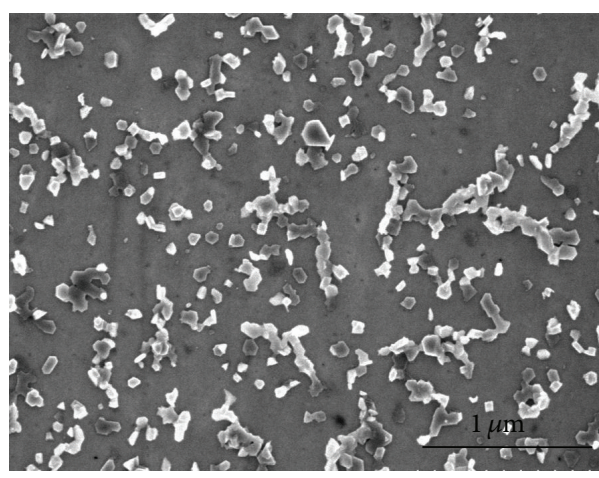

(c)

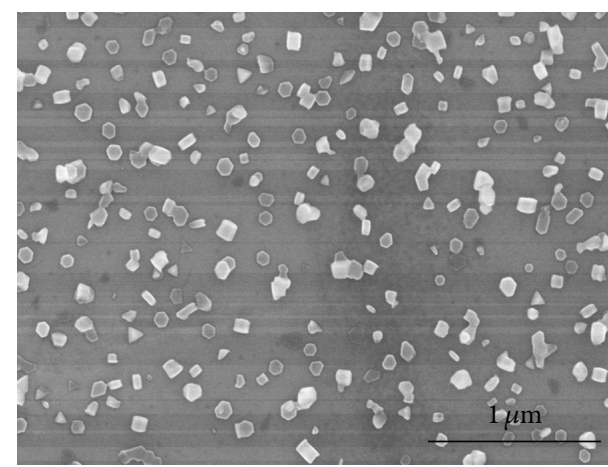

(b)

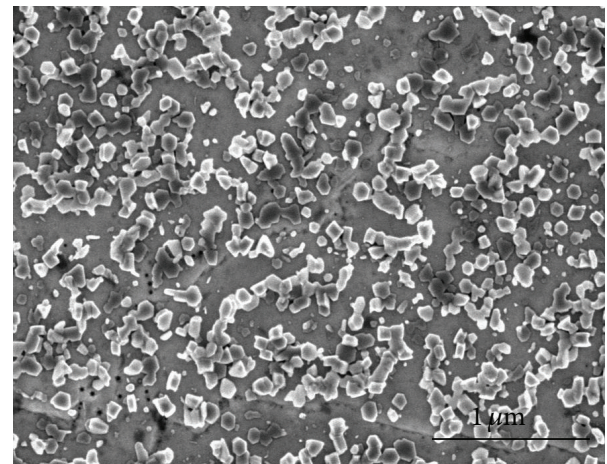

(d)

Figure 8: SEM images of $\mathrm{Zn}$ deposits on GaN electrode with an applied potential of $-1.4 \mathrm{~V}$. The deposition time was (a) 10, (b) 20, (c) 40, and (d) $80 \mathrm{~s}$.

Program of Jiangsu Province (no. BE2015073), and the Chinese Academy of Sciences.

\section{References}

[1] A. Gomes and M. I. Da Silva Pereira, "Pulsed electrodeposition of $\mathrm{Zn}$ in the presence of surfactants," Electrochimica Acta, vol. 51, no. 7, pp. 1342-1350, 2006.

[2] P. Díaz-Arista, Z. I. Ortiz, H. Ruiz, R. Ortega, Y. Meas, and G. Trejo, "Electrodeposition and characterization of $\mathrm{Zn}-\mathrm{Mn}$ alloy coatings obtained from a chloride-based acidic bath containing ammonium thiocyanate as an additive," Surface and Coatings Technology, vol. 203, no. 9, pp. 1167-1175, 2009.

[3] Z. F. Lodhi, J. M. C. Mol, W. J. Hamer, H. A. Terryn, and J. H. W. De Wit, "Cathodic inhibition and anomalous electrodeposition of Zn-Co alloys," Electrochimica Acta, vol. 52, no. 17, pp. 54445452, 2007.

[4] A. Gomes, A. S. Viana, and M. I. Da Silva Pereira, "Potentiostatic and AFM morphological studies of Zn electrodeposition in the presence of surfactants," Journal of the Electrochemical Society, vol. 154, no. 9, pp. D452-D461, 2007.

[5] A. Gomes and M. I. D. S. Pereira, "Zn electrodeposition in the presence of surfactants: part I. Voltammetric and structural studies," Electrochimica Acta, vol. 52, no. 3, pp. 863-871, 2006.

[6] R. C. M. Salles, G. C. G. De Oliveira, S. L. Díaz, O. E. Barcia, and O. R. Mattos, "Electrodeposition of $\mathrm{Zn}$ in acid sulphate solutions: PH effects," Electrochimica Acta, vol. 56, no. 23, pp. 7931-7939, 2011.
[7] G. Trejo, Y. Meas, and P. Ozil, "Nucleation and growth of zinc from chloride concentrated solutions," Journal of the Electrochemical Society, vol. 145, no. 12, pp. 4090-4097, 1998.

[8] J. Yu, H. Yang, X. Ai, and Y. Chen, "Effects of anions on the zinc electrodeposition onto glassy-carbon electrode," Russian Journal of Electrochemistry, vol. 38, no. 3, pp. 321-325, 2002.

[9] D. S. Baik and D. J. Fray, "Electrodeposition of zinc from high acid zinc chloride solutions," Journal of Applied Electrochemistry, vol. 31, no. 10, pp. 1141-1147, 2001.

[10] E. Michelakaki, K. Valalaki, and A. G. Nassiopoulou, "Mesoscopic Ni particles and nanowires by pulsed electrodeposition into porous Si," Journal of Nanoparticle Research, vol. 15, no. 4, article 1499, pp. 211-214, 2013.

[11] M. L. Calegaro, M. C. Santos, D. W. Miwa, and S. A. S. Machado, "Microgravimetric and voltammetric study of $\mathrm{Zn}$ underpotential deposition on platinum in alkaline medium," Surface Science, vol. 579, no. 1, pp. 58-64, 2005.

[12] H. Y. Yang, X. W. Guo, X. B. Chen et al., "On the electrodeposition of nickel-zinc alloys from a eutectic-based ionic liquid," Electrochimica Acta, vol. 63, pp. 131-138, 2012.

[13] J. R. I. Lee, R. L. O’Malley, T. J. O’Connell, A. Vollmer, and T. Rayment, "X-ray absorption spectroscopy characterization of $\mathrm{Zn}$ underpotential deposition on $\mathrm{Au}\left(\begin{array}{lll}1 & 1 & 1\end{array}\right)$ from phosphate supporting electrolyte," Electrochimica Acta, vol. 55, no. 28, pp. 8532-8538, 2010.

[14] R. Özdemir and I. H. Karahan, "Electrodeposition and properties of $\mathrm{Zn}, \mathrm{Cu}$, and Cul-x $\mathrm{Znx}$ thin films," Applied Surface Science, vol. 318, pp. 314-318, 2014. 
[15] D.-H. Kim, W. C. Lim, J.-S. Park, and T.-Y. Seong, "Highly thermally stable $\mathrm{Pd} / \mathrm{Zn} / \mathrm{Ag}$ ohmic contact to Ga-face p-type GaN," Journal of Alloys \& Compounds, vol. 588, no. 5, pp. 327331, 2014.

[16] W.-S. Yum, J.-W. Jeon, J.-S. Sung, and T.-Y. Seong, "Highly reliable $\mathrm{Ag} / \mathrm{Zn} / \mathrm{Ag}$ ohmic reflector for high-power GaN-based vertical light-emitting diode," Optics Express, vol. 20, no. 17, pp. 19194-19199, 2012.

[17] P. G. Eliseev, P. Perlin, J. Furioli, P. Sartori, J. Mu, and M. Osiński, "Tunneling current and electroluminescence in InGaN: $\mathrm{Zn}, \mathrm{Si} / \mathrm{AlGaN} / \mathrm{GaN}$ blue light emitting diodes," Journal of Electronic Materials, vol. 26, no. 3, pp. 311-319, 1997.

[18] Y. Lei, J. Xu, and K. Zhu, "A GaN-based LED with perpendicular structure fabricated on a $\mathrm{ZnO}$ substrate by MOCVD," Journal of Display Technology, vol. 9, no. 5, pp. 377-381, 2013.

[19] A. Nakajima, K. Takao, and H. Ohashi, "GaN power transistor modeling for high-speed converter circuit design," IEEE Transactions on Electron Devices, vol. 60, no. 2, pp. 646-652, 2013.

[20] A. V. Babichev, H. Zhang, P. Lavenus et al., "GaN nanowire ultraviolet photodetector with a graphene transparent contact," Applied Physics Letters, vol. 103, no. 20, Article ID 201103, 2013.

[21] M. Hirayama, Y. Ueta, T. Konishi, and S. Tsukamoto, "Nanoclustered Pd catalysts formed on GaN surface for green chemistry," Journal of Crystal Growth, vol. 323, no. 1, pp. 150-153, 2011.

[22] S.-Y. Chiu, H.-W. Huang, T.-H. Huang et al., "Comprehensive investigation on planar type of $\mathrm{Pd}-\mathrm{GaN}$ hydrogen sensors," International Journal of Hydrogen Energy, vol. 34, no. 13, pp. 5604-5615, 2009.

[23] T. Konishi, Y. Ueta, M. Hirayama, N. Nishiwaki, and S. Tsukamoto, "S-termination effects for the catalytic activities of Pd on GaN(0001) surfaces," Applied Surface Science, vol. 258, no. 21, pp. 8334-8337, 2012.

[24] Y. Zhao, F.-X. Deng, L.-F. Hu, Y.-Q. Liu, and G.-B. Pan, "Electrochemical deposition of copper on single-crystal gallium nitride(0001) electrode: nucleation and growth mechanism," Electrochimica Acta, vol. 130, no. 4, pp. 537-542, 2014.

[25] Y. Li, Y. Zhao, G.-B. Pan, Z.-H. Liu, G.-L. Xu, and K. Xu, "Preparation of platinum nanoparticles on $n-\mathrm{GaN}(0001)$ substrate by means of electrodeposition," Electrochimica Acta, vol. 114, pp. 352-355, 2013.

[26] R. Morita, T. Narumi, and N. Kobayashi, "Simultaneous monitoring of hydrogen generation and flat-band potential by measuring bias dependent photoluminescence of n-type GaN/electrolyte system," Japanese Journal of Applied Physics, vol. 45, no. 4, pp. 2525-2527, 2006.

[27] G. Oskam, J. G. Long, A. Natarajan, and P. C. Searson, "Electrochemical deposition of metals onto silicon," Journal of Physics D: Applied Physics, vol. 31, no. 16, pp. 1927-1949, 1998.

[28] L. E. Morón, A. Méndez, F. Castañeda et al., "Electrodeposition and corrosion behavior of $\mathrm{Zn}$ coatings formed using as brighteners arene additives of different structure," Surface and Coatings Technology, vol. 205, no. 21-22, pp. 4985-4992, 2011.

[29] P. Díaz-Arista, Y. Meas, R. Ortega, and G. Trejo, "Electrochemical and AFM study of $\mathrm{Zn}$ electrodeposition in the presence of benzylideneacetone in a chloride-based acidic bath," Journal of Applied Electrochemistry, vol. 35, no. 2, pp. 217-227, 2005.

[30] P. Delahay and T. Berzins, “The Internationale FunkAustellung in Berlin has got more electronic innovations that you can point a remote control at David Phelan Picks the very latest in audiovisual entertainment," Journal of the American Chemical Society, vol. 75, no. 10, pp. 2486-2493, 1953.
[31] P. M. Vereecken, F. V. Kerchove, and W. P. Gomes, "Electrochemical behaviour of (100) GaAs IN copper(II)-containing solutions," Electrochimica Acta, vol. 41, no. 1, pp. 95-107, 1996. 

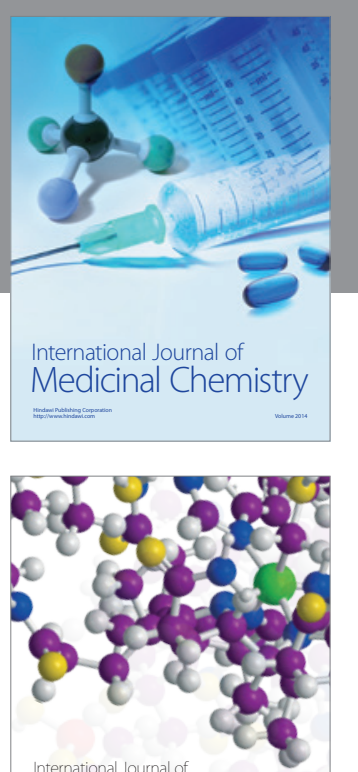

Carbohydrate Chemistry

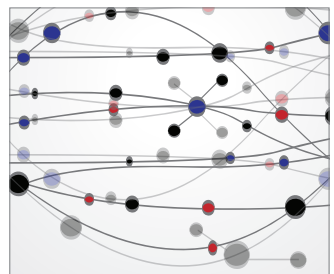

The Scientific World Journal
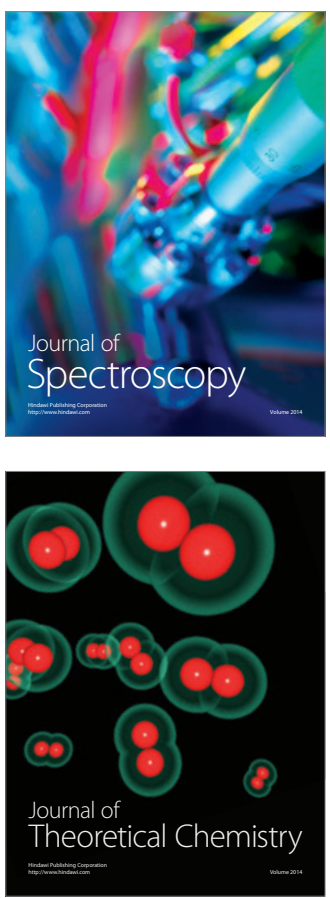
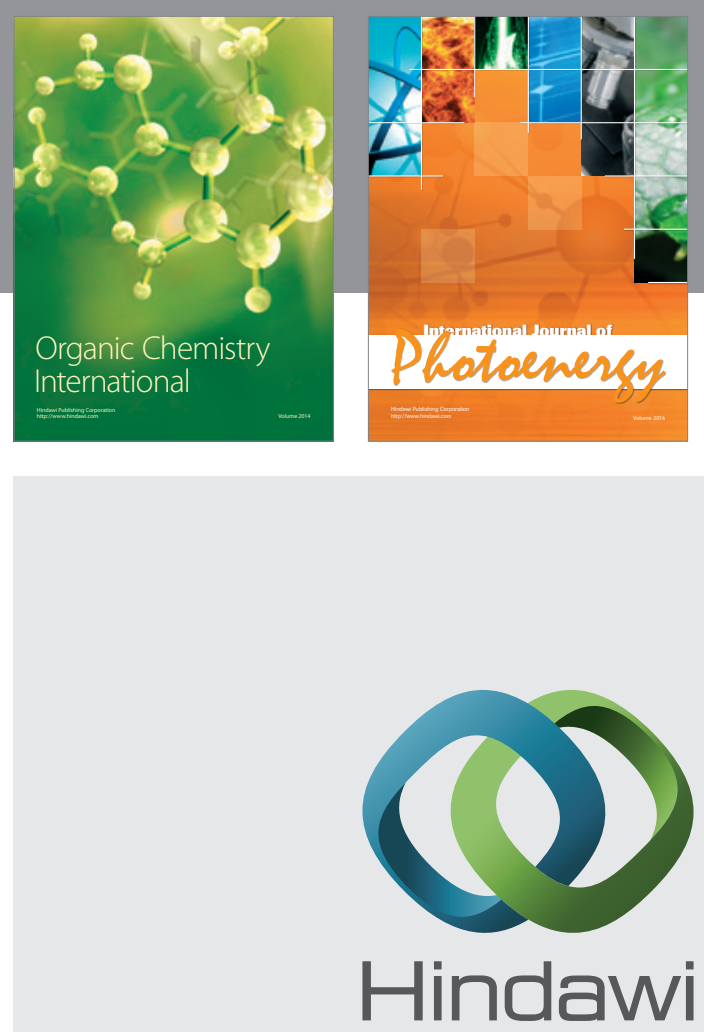

Submit your manuscripts at

http://www.hindawi.com

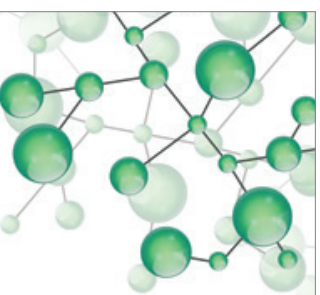

International Journal of

Inorganic Chemistry

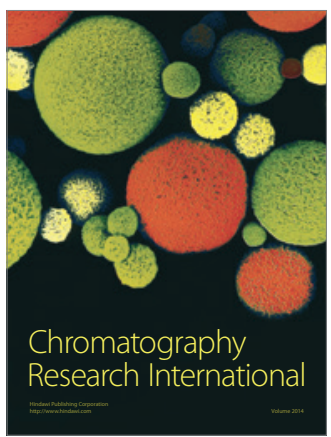

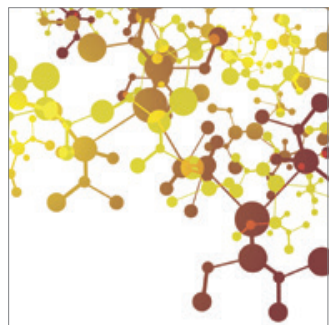

Applied Chemistry
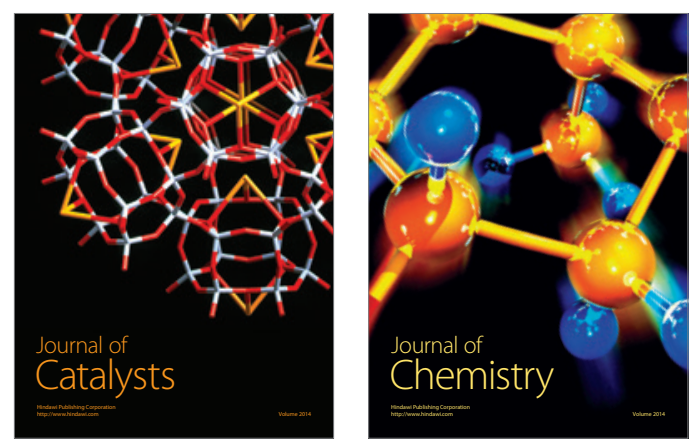
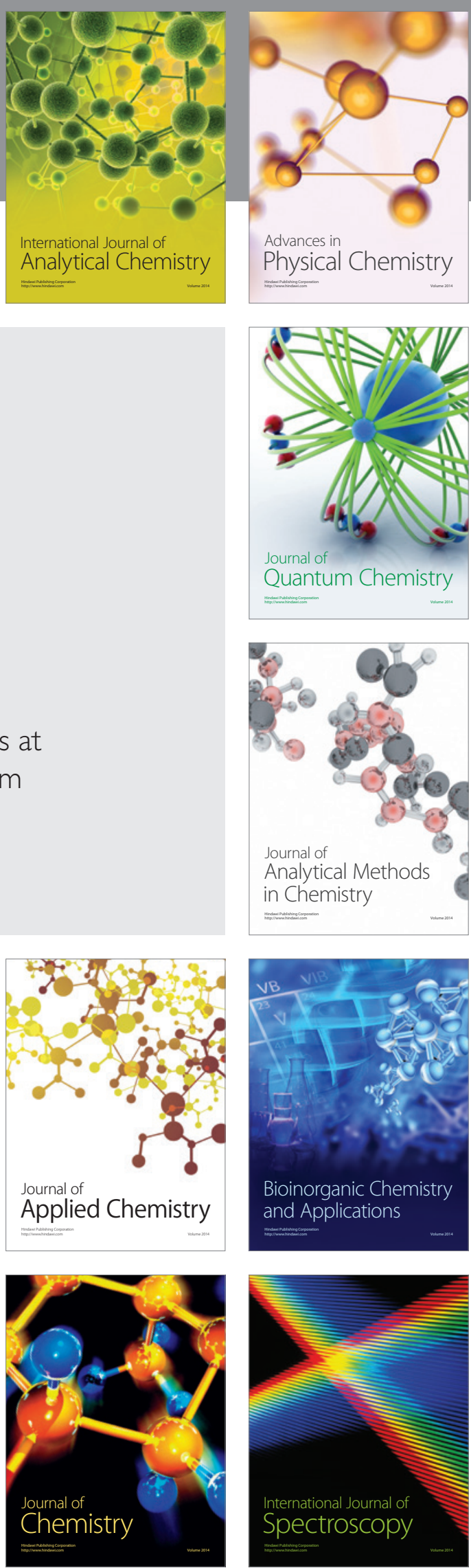